\title{
To Understand the Health Care Seeking Pathways for the HIV Positive Patients attending Rehabilitation Centre
}

\author{
Dr. Om Prakash ${ }^{1}$, Dr.Pushpa Prakash ${ }^{2}$ \\ ${ }^{1}$ (Senior Resident Department of PMR \&Orthopaedics, Nalanda Medical College and Hospital Patna,India) \\ ${ }^{2}$ (Medical Officer, NGR Hospital Patna, India)
}

\begin{abstract}
The epidemic of human Immunodeficiency virus (Retrovirus) that causes Acquired Immunodeficiency Syndrome has emerged as a serious public health problem in many parts of the World, which breaks down body's immune system leading to life threatening opportunistic infections and unusual malignancies. In which gender based difference in the health seeking behaviour has significantly precipitate to it. Rationale: Women's are much more vulnerable and likely to get discriminated with HIV infection, having so many reasons behind it than men, who plays dominant role in the society. So, there is need of gender based research to go through the health seeking behaviour of the HIV positive patients to understand the discrimination. This study also helps in policy making for National AIDS Control Program. Aims \& Objectives: To study the health seeking behaviour of HIV positive case and impact ofgender discrimination over it. Methods: This study was based on qualitative data collection of 25 HIV positive patients conducted between the April 2012 to June 2012 at Rajendra Memorial Research Institute (ICMR) Patna Bihar. 25 HIV positive patients (More than 13 years of age) who visit the Rehabilitation centre during the study period. A semi-structured-interview topic guide was used for $H I V$ positive patients with the help of the expert counsellors. In-depth interview of some randomly selected patients were arranged to collect the detailed information on health seeking behaviour at ART rehabilitation centre RMRI Patna with prior information, written and verbal consent. Prior permission will be taken from Ethical committee of Indian Institute of Public Health Delhi. Results: This study included 16 (64\%) females and $9(36 \%)$ males HIV positive cases who visited ART Rehabilitation RMRI Patna. Half of the patients (52\%) were 31-40 years of age group among boththe genders. Majority of female patients (75\%) were housewives. While majority of males were involvedin high risk occupations like Truck drivers (44.44\%). Majority of female patients $(68.75 \%)$ were illiterate. While majority of male patients (44.44\%) were educated up to 7 'th standard. $92 \%$ were of low incomegroup ranges Rs.2000-8000 per month. Health issues (60\%) were the main issue of HIV positive patientto come at ART centre RMRI Patna. Females were more vulnerable (60\%). Out of 25 patient $88 \%$ patients were referred by ICTC, doctors (private \& government), NGO. First symptoms in HIVpositive patient noticed by them had history of single or multiple symptoms during initial phase, inwhich single symptom was $40 \%$ and with more than one complain was $60 \% .77 .8 \%$ patients initiallyhave been taking home remedies, from quacks, in which $33.33 \%$ were males and $43.75 \%$, were females.Study shows that $60 \%$ patient contacted Government doctors, private doctors, NGO initially, in which $20 \%$ were females as compared to $24 \%$ males.
\end{abstract}

Keywords:HIV/AIDS, RMRI, Health Seeking Behaviour.

\section{Introduction:}

The epidemic of human Immunodeficiency virus(Retrovirus) that causes Acquired Immunodeficiency Syndrome hasemerged as a serious public health problem in many parts of the World, which breaks down body's immune system leading to life threateningopportunistic infections and unusual malignancies, in which gender based difference in the Health Seeking Behaviours has significantly precipitate to it. The victim once infected that will persist for whole life. AIDS is recognized as an emerging disease only in the early 1980's. This mysterious illness has rapidly established itself throughout the world as a pandemic. The latest statistics of global HIV \& AIDS epidemic were published by UNAIDS WHO \& UNICEF in November $2011 \&$ refer to the end of 2010. People living with HIV/AIDS estimate 34 million (range 31.6- 35.2million) in which proportion of adult women percentage estimates 50 (range $47-53$ ), children is 3.4 million (range $3.0-3.8$ million), newly infected children is 390,000 (range 340,000 -450,000) \& almost 1.8 million (range $1.6-1.9$ million) have lost their lives. The number of people living with HIV rose from around 8 million in 1990 to 34 million by the end of 2010[1]. In Sub Saharan Africa, young women (aged 15 -24 years )are infected more frequently than young men .In 2001 , the estimated infection rates for young women were 6-11 per cent compared to $3-6$ per cent for young men[2] .FOCUS OF GENDER IN HIV / AIDS: - At the end of 2006, 37.2

Million adult living with HIV AIDS[3] .In which 17.7 million nearly 50per cent were women. Whereas report of 2011 proportion of adult womenpercentage estimates 50 (range 47 - 53). Rather thanrepresenting equitybetween sexes the one to one ratio of male to female HIV infectionsdemonstrates the way in which gender 
inequalities affects HIV /AIDSincidence rates. Since the HIV /AIDS began more than 30 years ago,infection rate among women have accelerated .Traditional gender rolesheld by many of the world's societies definitely affects the health seekingbehaviours of women , which continued spread of HIV ;particularly frommen to women . In most societies, women also carry a disproportionateamount of the burden of caring for family members living with HIV/AIDS and experience the brunt of the stigma associated with HIVinfection. Correcting the gender imbalance that contributes to and isexacerbated by the HIV /AIDS pandemic will depend upon improvingwomen's social and economic status, and increasing men's responsibilityfor HIV prevention and care, so as to provide better health care access. Unequal social roles and vulnerability to men's demands prevent womento have better health care access . Number of facts associated with HIVtransmission like illiteracy, unemployment, gender discrimination, poorhealth infrastructure etc. stimulates already prevalent stigma related to HIV/AIDS. All these factors such as gender roles,economic, cultural, socialfactors and stigma related to HIV /AIDS are likely to influence themotivation of HIV infected people to practice safe sex[4] .NACO reportedfor HIV /AIDS in India the total number of people living with HIV /AIDS is estimated at 23.9 lac (range 19.3 -30.4 lac) in2009 .Of all HIV infections,39 per cent (9.3 lac) are among women .Nash Ojanuga and Gilbert (1992) systematized the obstacles which women face into four categories: -(1) CULTURAL BARRIERS - Social status of women which situates them in socially inferior position. (2) EDUCATION BARRIERS -Women having less access to education. (3) INSTITUTIONAL BARRIERS -Unequal treatment by health providers. (4)ECONOMIC BARRIERS -Different access to resources [5]. Available studies, scientific and research papers, grey literature, reports and other primary and secondary sources of information were reviewed. Internet and web-based research Pub med, Google Scholar, Mozilla and others will be extensively used to find the relevant materials on the following provisions for this review. A review by USAID Health policy initiative, Health Seeking behaviour in rural Uttar Pradesh: Implications for HIV prevention, care,\& treatment 2009 shows that uptake of preventive health services is low in the state, especially among rural and uneducated women. Low literacy and lack of awareness about services, schemes, and entitlement low states of women, poverty, and cultural factors, lack of family support are among the crucial factors that determine the Health Seeking behaviour in the state[6] .Another study of Health Seeking behaviour of persons with HIV / AIDS in Ghana by Kofi Awusabo-Asare and John K. Anarfi et all (1997) found that 141 sero positive patients, with a sex ratio of 40 males per 100 females[7].

\section{Management Challenges:}

This is the sensitive topic. Due to social stigma no one wants to disclose his/ her status to anyone, even though the most nearest and dearest personnel are not disclose his/her status to each other. In the society no one give sympathy to these patients because mostly know these disease are of bad character habitants. During interview being a sensitive regarding the sexual relationship with many partners some times its very difficult for interviewer \& participants how to interact with each other. Challenges faced by participants during the course of ART at centre due to lack of human resources, due to overcrowding (work load) human resources are not tackling these participants or patients in a proper guidelines provided by WHO/NACO. The challenges faced by patients / participants at the home, workplace first they deprived from their work due to lack of physical power, unable to earn so much for livelihood. As a result his/her health deteriorates continuously. This vicious cycle persist till his her death. Health Seekingbehaviour changed with their partner after three or four sorts of counselling given by the counsellors. The illiteracy also plays a great role in the management of these patients.

\section{Rationale:}

Women's are much more vulnerable and likely to getdiscriminated with HIV infection, having so many reasons behind it than men, who plays dominant role in the society. So, there is need of gender based research to go through the health seeking behaviour of the HIV positive patients to understand the discrimination. This study also helps in policy making for National AIDS Control Program.

\section{Aims and Objectives:}

To study the health seeking behaviour of HIV positive case and impact of gender discrimination over

it.

\section{Methodologies:}

This study was based on qualitative data collectionof $25 \mathrm{HIV}$ positive patients conducted betweenApril 2012 to June 2012 at Rajendra Memorial Research Institute (ICMR) Patna Bihar. The totalnumbers of HIV positive patients (More than 13 years of age) were visited at RMRI Rehabilitation centre Patna during the study period. A semi structured -interview topic guide was used for HIV positive patients with the help of expert counsellors. In-depth interview of some randomly selected patients was arranged to collect the detailed information's in a private room through health seeking behaviour pathway at RMRI Rehabilitation centre with 
prior information obtaining written and verbalconsent. In this regard proper care was taken to maintain theconfidentiality. Prior permission was taken from Ethical committee ofIndian Institute of Public Health Delhi to carry out this dissertation. Thefollowing criteria were adopted for inclusion of HIV positive patientsattending ART rehabilitation centre for which this protocol wasmaintained: (1)16 females and 9 males of HIV positive patient $>13$ yearsof age. (2) Patients were HIV positive as per NACO guidelines. (3)Patients, who voluntarily took part in the interview, visited ARTRehabilitation Centre at RMRI Patna after HIV infection. (4) 2 Counsellorsand 3 Doctors of ART Rehabilitation centre RMRI Patna were included inthis study.

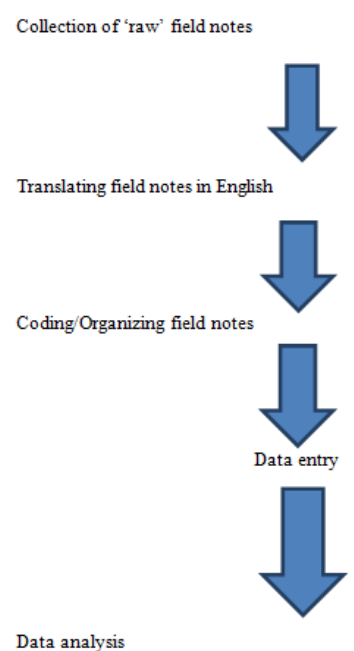

Fig 1: The following steps were taken for documentation and data managementafter data collection.

5. 1 Limitation of study: (1) we have covered only ART Rehabilitation centres RMRI (ICMR) Patna. (2) The duration of study was of two and half months period only in which 25 IDI concerned to health seeking behaviour of HIV positive patients attending ART Centre RMRI (ICMR) Patna and 5 IDI of those Counsellors\& Doctors regarding their experiences with HIV positive patients were conducted.

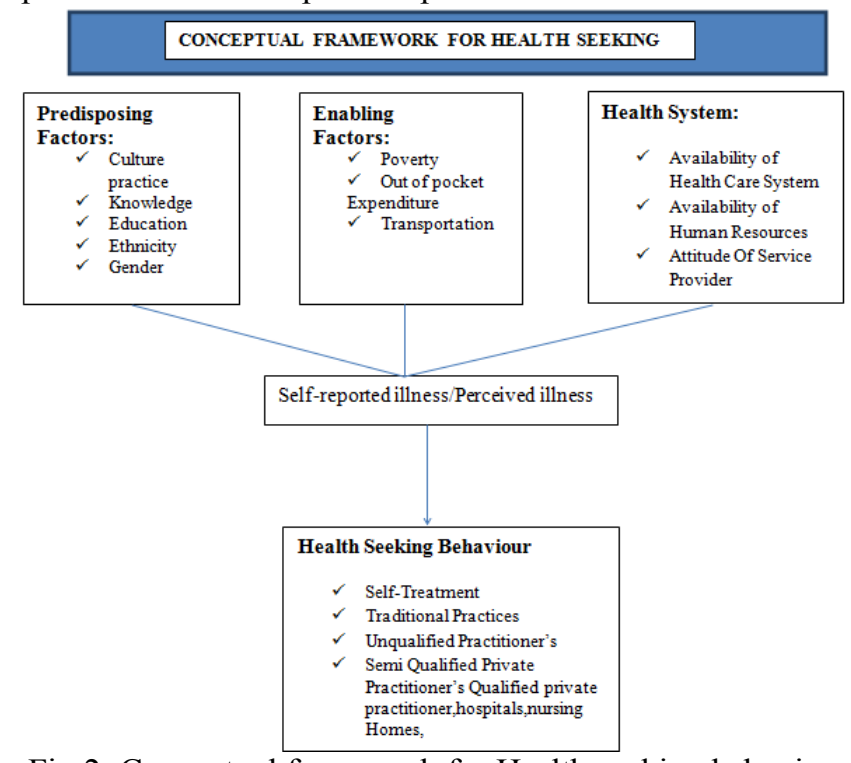

Fig 2: Conceptual framework for Health seeking behaviour

\subsection{Pathway of health seeking behaviour of HIV positive patient:}

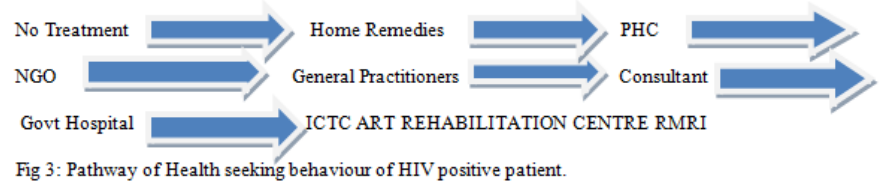

Figure: - 1 Shows the conceptual model used in this study. In specifyingthe various factors influencing healthseeking behaviour, we relied on abehavioural model and its subsequent modification [8][9][10][11][12][13]. 
The modifiedVersion of the model has been successfully applied in the study of healthservices utilization in developing countries [12][13]. This model proposes thathealth-seeking behaviour is a function of three sets of individualCharacteristics: predisposing factors, enabling factors, and Health system. The actual seeking of health services is assumed to be a sequential andconditional function of the individual's predisposition to use healthServices, their perceived need to use them, and their ability to obtain theservices. Some variables may belong in more than one of these categories.In such a case, we made an arbitrary classification for the analysis andPresentation of our findings. The predisposing factors (i.e., Culture,practice, knowledge, education, gender and ethnicity), and enabling factors(i.e., Poverty, Out of pocket expenditure and Transportation) areconsidered as independent variables affecting health-seeking behaviour. The enabling factors are those by which individuals have the means thatpermit them to obtain health services. Finally, although predisposing andEnabling factors are necessary for the use of health services, they are notsufficient for actual use; the actual use is triggered by the need duringprenatal and postnatal stages, childbirth, or general illness [8]. In the presentStudy, we explored the extent to which the predisposing and enablingfactors contributed to any differences in health-seeking behaviour.

\section{Results:-}

This study included $16(64 \%)$ females and $9(36 \%)$ malesHIV positive cases who visited ART Rehabilitation RMRI Patna. Half ofthe patients (52\%) were 31-40 years of age group followed by 21-30 years $\& 41-50$ years $(20 \%)$ each respectively, whereas $14-20$ years \& 51-60 years $(4 \%)$ each respectively among both the genders.
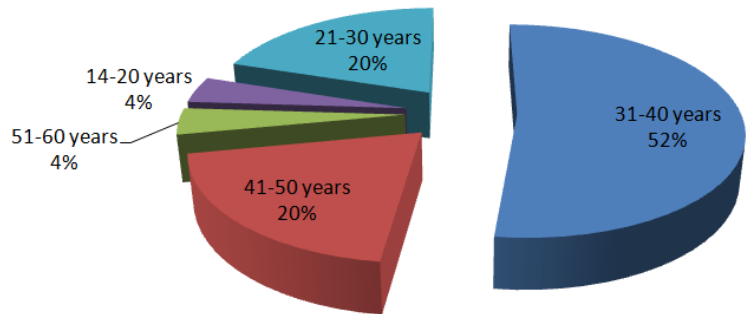

Fig. 4: \% of HIV positive pt. visited ART Rehabilitation Centre (Age group wise)

Almost $96 \%$ of HIV positive patients were married with significantlyhigher numbers of females $(66.66 \%)$ as compare to males $(33.33 \%)$. Whereas $4 \%$ i.e. a single patient was unmarried.

\section{\% of Marrital Status of HIV positiv Pt. visited}

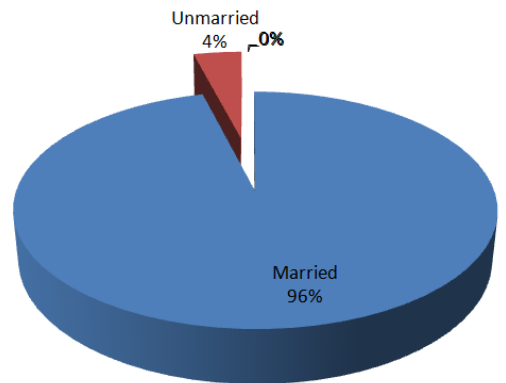

Fig. 5: \% of Marital Status of HIV positive Pt. visited ART Rehabilitation Centre

Majority of female patients (75\%) were housewives followed by $12.5 \%$ oflabour workers and $6.25 \%$ each of Bidi workers (Tobacco) and Teachers.

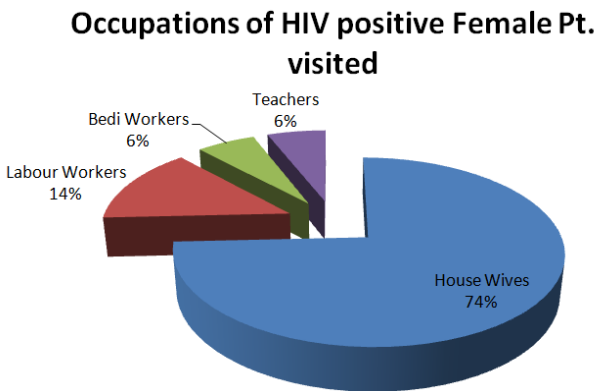

Fig 6: Occupations of HIV positive Female Pt. visited ART Rehabilitation Centre In \% 
While majority of males were involved in high risk occupations like Truck drivers (44.44\%) followed by Grocery shopkeeper (22.22\%), farmer, labour \& private job (11.11\%) of each respectively.

\section{Occupations of HIV positive Male pt. visited}

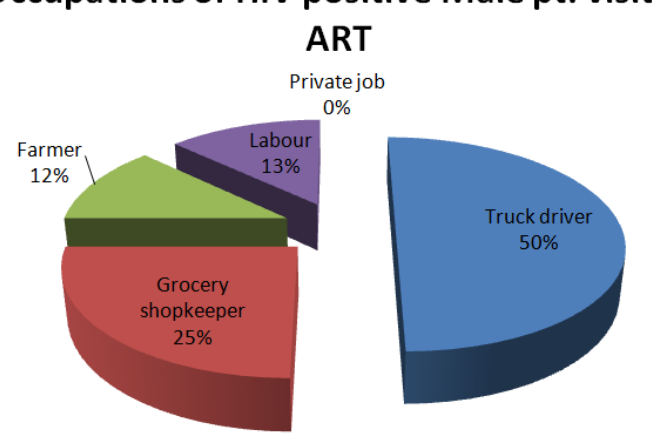

Fig.7: Occupations of HIV positive Male pt. visited ART Rehabilitation Centre in \%

So far as literacy point of view is concerned majority of female patients $(68.75 \%)$ were illiterate followed by $18.75 \%$ patients were educated up to $10^{\prime}$ th standard, $12.5 \%$ up to $7^{\prime}$ th standard. While majority of male patients(44.44\%) were educated up to $7^{\prime}$ th standard followed by (33.33\%) up to10'th standard, whereas $11.11 \%$ were illiterate \& up to $12^{\prime}$ th standard eachrespectively. Out of the total patients the socio-economic condition of the $92 \%$ was of low income (ranges Rs.2000-8000) per month whereas $8 \%$ were of average income (ranges $>8000-15000$ ) per month among both thegenders. All HIV positive patients had undergone CD4 count testing ofwhom $64 \%$ were females and $36 \%$ were males. In majority of cases about $2-3 \%$ cases had history of ADR in the form of nausea, vomiting, anaemia,skin rashes. Health issues $(60 \%)$ were the main issue followed bycombination of health issue \& social issue ( $40 \%$ ) of HIV positive patient tocome at ART centre RMRI Patna. As regardsvulnerability issue related togenders, females were more vulnerable $(60 \%)$ than males $(40 \%)$. Spousedisclosure issue concerned with constant use of contraception was $60 \%$ followed by high risk behaviour $(40 \%)$ with no gender difference as statedby expert counsellors\& doctors of ART centre RMRI Patna. Out of 25 patient $88 \%$ patients were referred by ICTC, doctors (private \&government), NGO and $12 \%$ of patients directly come to RMRI Patna by their family members as their husbands were known case of HIV positive patient. First symptoms in HIV positive patient noticed by them had history of single or multiple symptoms during initial phase, in which single symptom was $40 \%$ and with more than one complain was $60 \%$. Majority of patients observed that fever (36\%), diarrhoea/loose motion (24\%), weakness $(20 \%)$, oral/vaginal candidiasis $(16 \%)$, cough \& itching all over the body (12\%) each respectively, anorexia (8\%) \& other symptoms was burning sensation all over the body, wound, indigestion, hematemesis, multiple joint pain, tuberculosis, vaginal discharge, weight loss. Out of 25 patients, 3 patients investigated due to their husbands were known HIV positive case. $77.8 \%$ patients initially have been taking home remedies, from quacks, in which $33.33 \%$ were males and $43.75 \%$, were females. Study shows that $60 \%$ patient contacted Government doctors, private doctors, NGO initially, in which $20 \%$ were females as compared to $24 \%$ males. The fact that males have better awareness towards their health than females. $12 \%$ patient consulted 1 health care provider, $24 \%$ consulted 2 health care providers, $44 \%$ consulted 3 health care providers, $16 \%$ consulted 4 health care providers \& $4 \%$ consulted 5 health care providers before reaching ICTC at RMRI Patna.

\section{$\%$ of Patient Consulted Number of health care}

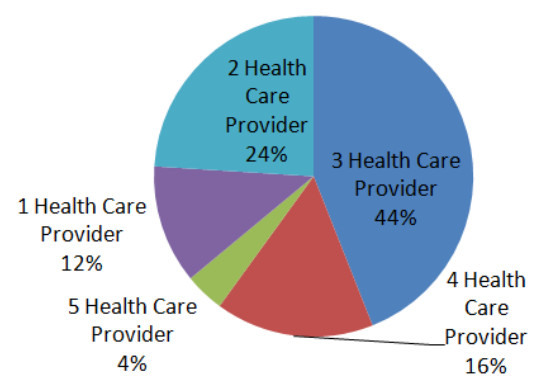

Fig 8: \% of Patient Consulted Number of health care providers

Out of 25 patients $44 \%$ patients had first health care contact within 3months, $20 \%<6$ months, $16 \%<12$ months of observing first sign \&symptoms. While $20 \%$ patients took $>12$ months to contact health careproviders. According to health care seeking pathway-initially44\% patients directly went to Quack, in which males were 
$12 \%$ \& females were $32 \%$ with the hope of getting appropriate treatment, but finally consulted Government Doctors / Private Doctors for their treatment. $12 \%$ patients directly went to ICTC, RMRI Patna for investigation andtreatment purpose. The probable reason for HIV infection was(1) multiple sexual contacts with different sexual partners,(2) husbands themselves were HIV patients and (3)due to blood transfusion. In which $36 \%$ were males and $54 \%$ were females. In majority of cases $72 \%$ were having multiple sexual contacts. Out of which $32 \%$ were males and $40 \%$ were females. Husbands responsible for HIV infection were $16 \%$ and $12 \% \mathrm{HIV}$ infection was due to blood transfusion.

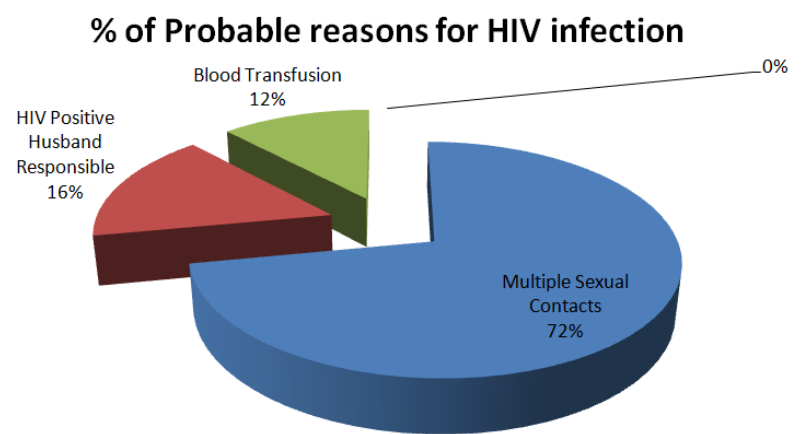

Fig 9: \% of Probable reasons for HIV infection

In context to types of problems faced by HIV positive patient at variouslevels-in which majority of cases $(60 \%)$ had no family problems butoutsiders do not know about their HIV status, followed by $20 \%$ had been facing financial problems but family was supportive, $16 \%$ had been facing problems in family and society whereas $4 \%$ i.e. a single was being hatred by others but no family problem.

\section{Discussion:}

Majority of females(32\%) initially contacted to quack as compared to male $(12 \%)$. This was because of the male dominance to their decision whereas female has to depend on others physically as well as economically. Male patients (24\%) had contacted Government hospital /private clinic initially for their health problems as compared to $(20 \%)$ females. Because of fear of social stigma related to HIV/AIDS \&belief of more privacy at private setup. The fact that males have better awareness towards their health than females. Half of the patients (52\%) were 31-40 years of age group. $96 \%$ of HIV positive patients were married. Mostly female patients (75\%) were housewives. Whereas males were Truck drivers (44.44\%). Majority of female patients $(68.75 \%)$ were illiterate. While in case of males $(44,44 \%)$ were educated up to 7 'Th standard. $92 \%$ was of low socioeconomic group, income ranges (Rs. 2000-8000) per months. Only 2-3\% cases had history of ADR. Females were more vulnerable $(60 \%)$ than males $(40 \%)$. Health issues $(60 \%)$ were the main issue of HIV positive patients. The commons first symptoms in HIV positive patients was fever (36\%) followed by diarrhoea/loose motion (24\%). Initially $24 \%$ males contacted government doctors/private doctors/NGOs as compared to $20 \%$ of females. The factthat males have better awareness towards their health than females. Inmajority of cases $44 \% \mathrm{HIV}$ positive patients consulted three healthcareproviders. $44 \%$ patients had first healthcare contact within three months. The health seeking behaviour is based on the type of culture, medicalfacilities available, medical pluralism and perspective of individual towardsdisease, sleekness and illness. But in the community other factors such asgeographical distance, connectivity, transportation, money, unequaldistribution of health facility, lack of resources etc. were found.Such factors play an important role in the choice people make to accesshealth care facilities. Moreover people tend to seek health care facilitiesonly when the disease progresses or else they prefer to seek cures fromunqualified practitioners who are more accessible and are cheaper.Purchase over the counter drugs is also very common in this study.

They have the impression that treating at home by unqualified practitioner is much cheaper and time and numbers of day's loss in their daily agriculture activities are also saved if treated at home.

Other reasons like proximity of the health-care facilities, availability ofresources were also other determining health-seeking behaviour. Finally the attitude of health care staff also one of factors which determined the health seeking behaviour of general public.

\section{Conclusion and Recommendation:}

A Study on Gender impact of HIV/AIDS in India shows that as compared to men, women have poorer access to healthcare. The financial constraint is also a big impediment for getting proper healthcare and it is more so for women [14]. Women may be more affected by stigma and discrimination than men because of social norms concerning acceptable sexual behaviour in women, and that's why women are often more economically vulnerable than men. Our study also reported number of economically vulnerable females. Gender 
differences in decision-making may also affect access to health facilities. Another report by centre for disease control and prevention shows that as far as possible, women would try to avoid going to the doctor, in particular, avoid getting hospitalized as the household routine is likely to be disrupted more by the sickness of women of the household. In households where both men and women are sick, more importance is given to get the man who is the breadwinner, the same may be the reason in our study as we found less number of women who accessed treatment. . Advocacy talks on HIV/AIDS control need to be delivered to employees of public and private institutions. Education regarding HIV/AIDS must be imparted through schools, hospital outreach programmes, existing government health programmes such as maternaland child health programmes and routine home visits, using suitablematerials. Posters\& Road shows held at different parts of India can behelpful in gathering crowd \& thereby spreading the message of risks about HIV/AIDS involved among the public. Another important milestone in achieving anti-AIDS campaigns can be achieved when different religious leaders pledge to support the initiative. Outreach programmes from hospitals can educate the community about the dangers of AIDS. The study of Gender based Health seeking behaviours is the vital way to control the spread of HIV/AIDS. So strengthen the health services with the focus on gender. So, there is need of awareness of all respect, prevention of transmission, adherence for ART drugs to take regularly. Follow-up of HIV positive patients strengthen through community care centre, out rich workers and by telephonic contacts. On the other hand medicalrehabilitation is an integral component of total health care delivery system. The present national scenario depicts that the persons with HIV \& AIDS infections invariably suffers with different spectrum of disabilities. With the introduction of United Nations Convention on rights of persons with disabilities in India \&in the process of reinforcing persons with disability Act 1995, it becomes rather imperative to protect \& insure the rights of persons with HIV/AIDS infections so that he/she can lead a respectable life in the society. Prevention, promotion, and rehabilitation are the major sectors \& pillars for providing / securing standard public health system. Ifpersons with HIV/AIDS infection are not medically rehabilitated, it would definitely have a bearing on public health issues significantly.

\author{
Abbreviations: \\ ADR - Adverse Drug Reaction \\ AIDS - Acquired Immunodeficiency Syndrome \\ ART - Anti Retroviral Therapy \\ ARV - Anti Retroviral Drug \\ GP- General Practitioner \\ HIV - Human Immunodeficiency Virus \\ HSB- Health Seeking Behaviour \\ ICMR- Indian Council Of Medical Research \\ ICTC - Integrated Counselling and Testing Centre \\ IDI-- In-depth Interview \\ GP - General Practitioner \\ $\mathrm{MO}$ - Medical Officer \\ NACO-National AIDS Control Organisation \\ NGO - Non Government Organisation \\ PHC - Primary Health Centre \\ PLHIV - People living with HIV \\ STD - Sexually Transmitted Disease \\ STI - Sexually Transmitted Infection \\ TB - Tuberculosis \\ UNAIDS - United Nations For Acquired Immunodeficiency Syndrome \\ UNDP - United Nations Development Program \\ UNICEF-United Nations International Children Emergency Fund \\ USAID-United States Agency For International Development \\ UTI- Urinary Tract Infection \\ VDRL - Venereal Disease Research Laboratory \\ WHO - World Health Organization
}

\title{
References:
}

[1] Avert international HIV and AIDS charity, source-WHO/UNAIDS/UNICEF2010,2011,Global.HIV/AIDS Response: Epidemic Update and health sector progress towards Universal access2011. Available at: www.avert.org/world star.htm

[2] Joint United Nations Program me on HIV/AIDS (UNAIDS) 2006,AIDS Epidemic update (English original) Available at: http://www.unaids.org/global report/documents/20101123 Global Report full en.pdf

[3] Joint United Nations Program me on HIV/AIDS (UNAIDS) 2000.Men and AIDS: A gendered Approach. Geneva: UNAIDS. Available at: www the body.com/unaids/men/contents. Html. Accessed on 1st August 2007. 


\section{To Understand the Health Care Seeking Pathways for the HIV Positive Patients attending}

[4] Population council (2007) "Reducing HIV risk behaviors among key populations by increasing community involvement and building social capital". Available at: www.popcouncil.org, Accessed on 1st November 2007.

[5] Ojanuga Nash, Gilbert (1992) Women's access to health care in Developing Countries. Social Science and Medicine V1. 35, August 1992:613.

[6] USAID Health policy initiative-Health Seeking Behavior in Rural Uttar Pradesh: Implications for HIV prevention, care and treatment, August 2009, 1-7. Available at: www.USAID.org.in/http://www.health policy initiative.com.

[7] Kofi Awusabo-Asore and John K.Anarfi, Health Seeking Behavior of persons with HIV/AIDS in Ghana; HealthTransition Review, Supplement to Volume 7, 1997,243-256.

[8] Andersen RM: A behavioural model of families' use of health services. Chicago, Illinois, USA: Centre for Health Administration Studies, University of Chicago; 1968.

[9]. Aday LA, Andersen RM: A framework for the study of access to medical care. Health Services Research 1974, 9:208-220.

[10] Andersen RM, Newman JF: Societal and individual determinants of medical care utilization in the United States.Milbank Memorial Fund Quarterly 1973, 51:95-124.

[11] Andersen RM: Revisiting the behavioural model and access to medical care: does it matter? Journal of Health and Social Behavior 1995, 36:1-10.

[12] Fosu GB: Childhood morbidity and health services utilization: Cross-national comparisons of user-related factors from DHS data. Social Science and Medicine 1994, 38:1209-1220.

[13] Subedi J: Modern health services and health care behavior: a survey in Kathmandu, Nepal.

[14] Matlin, S. \& Spence, N. (2001) "The Gender Aspects of the HIV/AIDS Epidemic." Presentation at the ExpertGroup Meeting on the HIV/AIDS Pandemic and Its Gender Implications, Windhoek, Namibia. Available at:www.un.org/womenwatch/daw/csw/hivaids/matlinspence.html. Accessed on 28th June 2012. 